\title{
ON THE KNUTH SEMI-FIELDS
}

\author{
D.R. HUGHES \\ Department of Mathematics \\ Westfield College \\ University of London \\ London \\ M.J. KALLAHER \\ Department of Mathematics \\ Washington State University \\ Pullman, Washington 99163 \\ U. S. A.
}

(Received May 28, 1979 and in revised form October 22, 1979)

ABSTRACT: We consider three of Knuth's four classes of semi-fields - Knuth [11] - namely, those having dimension 2 over a nucleus and show that the autotopism group is solvable (Corollary 4.12). This generalizes a result of Hughes [5]. We also show that for semi-fields of dimension 2 over a nucleus, the number of pairwise non-isomorphic isotopic images is at least 5 (Corollary 5.1.1). This generalizes a result in [10].

KEY WORDS AND PHRASES : Semi-fields, autotopism, determinant group, Knuth semifields.

1980 MATHEMATICS SUBJECT CLASSIFICATION CODES: 50005, 05B25, $27 E 05$. 


\section{INTRODUCTION.}

The central purpose of this article is to investigate the autotopism groups of one class of Knuth semi-fields. These semi-fields are defined as follows. Let $F$ be a finite field, let $S=F \times F$, let $\sigma$ be a non-identity automorphism of $F$, let $\tau=\sigma^{-1}$, and choose $f, g \in F$ such that $y^{\sigma+1}+g y-f \neq 0$ for all $y \in F$. (If $F$ is not a prime field, then there always exists $\sigma, f, g$ such that $\mathrm{y}^{\sigma+1}+\mathrm{gy}-\mathrm{f} \neq 0$ for all $\mathrm{y} \in \mathrm{F}$. See Knuth [11,p. 214].) Taking componentwise addition, the set $\mathrm{S}$ is a semi-field under any one of the following multiplications:

$$
\begin{array}{ll}
K(u): & (a, b)(c, d) \equiv\left(a c+b^{\sigma} d^{2} f, b c+a^{\sigma} d+b^{\sigma} d^{\sigma} g\right) \\
K(l): & (a, b)(c, d) \equiv\left(a c+b^{\sigma} d f, b c+a^{\sigma} d+b^{\sigma} d g\right) \\
K(r): & (a, b)(c, d) \equiv\left(a c+b^{\tau} d^{2} f, b c+a^{\sigma} d+b d^{\tau} g\right) \\
K(m): & (a, b)(c, d) \equiv\left(a c+b^{\tau} d f, b c+a^{\sigma} d+b d g\right)
\end{array}
$$

If $\mathrm{N}_{\mathrm{r}}, \mathrm{N}_{\mathrm{m}}, \mathrm{N}_{\ell}$ are the right, middle, and left nuclei, respectively, of $\mathrm{S}$ then the classes $K(\ell), K(r), K(m)$ are characterized by the following statement: A semi-field $S$ is of type $K(i)$ over the field $F$ if and only if (a) $N_{j}=F$ for $j \in\{r, m, l\}-\{i\}$ and (b) $S$ has (vector space) dimension 2 over F. (See Knuth [11].) The class $K(\ell)$ was discovered by Hughes and Kleinfeld [6] and Hughes [5] investigated the autotopism groups of such semi-fields with the principal result being that they are solvable. The semi-fields in $K(r)$ are obtained from the semi-fields of $K(l)$ by duality. As of yet the semi-fields in $K(m)$ have not been investigated. We do so in this article and show that Hughes's result for the class $\mathrm{K}(\ell)$ essentially holds also for the class $\mathrm{K}(\mathrm{m})$. (See Corollary 4.1.2)

Our proof of Corollary 4.1.2 is based upon the work of section 3. This section is based upon unpublished work of M. V. D. Burmeister, and the main result (Theorem 3.4) is a generalization of his techniques. In section 5, we extend a result of the article [10] to semi-fields having dimension 2 over one 
of their nuclei. Specifically, we show that such semi-fields must have at least 5 isotopic, but pairwise non-isomorphic, images.

We assume the reader is familiar with the theory of projective and affine planes as exhibited in [7].

\section{PRELIMINARIES.}

Throughout this section $S=\langle S, t, \cdot\rangle$ will be a finite semi-field of order $\mathrm{p}^{\mathrm{s}}$, where $\mathrm{p}$ is a prime, and $\mathrm{N}_{\mathrm{r}}, \mathrm{N}_{\mathrm{m}}, \mathrm{N}_{\ell}$ are respectively, the right, middle, and left nucleus of $\mathrm{S}$. Furthermore, $\mathrm{G}$ is the group of autotopisms of the semifield $S$; thus $G$ consists of all triples $\varphi=\left(\varphi_{1}, \varphi_{2}, \varphi_{3}\right)$ of bijective additive mappings of $\mathrm{S}$ with

$$
\left(\mathrm{x} \varphi_{1}\right)\left(\mathrm{y} \varphi_{2}\right)=(\mathrm{xy}) \varphi_{3} \text { for } \text { all } \mathrm{x}, \mathrm{y} \in \mathrm{S},
$$

and the operation in $G$ is componentwise composition. We have the following information.

LEMMA 2.1: Let $\mathrm{S}$ be a finite semi-field of order $\mathrm{p}^{\mathrm{s}}$, where $\mathrm{p}$ is a prime, and let $\mathrm{N}_{\mathrm{m}}$ be the middle nucleus of $\mathrm{S}$ having order $\mathrm{p}^{\mathrm{t}} \mathrm{m}$, and let $\mathrm{G}$ be the autotopism group of $\mathrm{S}$. The following statements hold:

(i) The semi-field $\mathrm{S}$ is both a left and right vector space over $\mathrm{N}_{\mathrm{m}}$ having dimension $\mathrm{d}=s \mathrm{t}_{\mathrm{m}}^{-1}$.

(ii) If $\varphi=\left(\varphi_{1}, \varphi_{2}, \varphi_{3}\right) \in \mathrm{G}$, then the mapping $\varphi_{\mathrm{m}}: \mathrm{N}_{\mathrm{m}} \rightarrow \mathrm{N}_{\mathrm{m}}$ given by $\mathrm{n} \varphi_{\mathrm{m}} \equiv\left(\mathrm{a}_{\varphi} \mathrm{nb}{ }_{\varphi}\right) \varphi_{3}$, when $\mathrm{a}_{\varphi}$ and $\mathrm{b}_{\varphi}$ are defined by $\mathrm{a}_{\varphi} \varphi_{1}=\mathrm{b}_{\varphi} \varphi_{2}=1$, is an automorphism of $\mathrm{N}_{\mathrm{m}}$.

(iii) If $\varphi=\left(\varphi_{1}, \varphi_{2}, \varphi_{3}\right) \in \mathrm{G}$ then $\varphi_{1}$ is a semi-linear transformation on $\mathrm{S}$ as a right vector space over $\mathrm{N}_{\mathrm{m}}$ (with companion automorphism $\left.\varphi_{\mathrm{m}}\right)$ and $\varphi_{2}$ is a semi-1inear transformation on $S$ as a left vector space over $\mathrm{N}_{\mathrm{m}}$ (with companion automorphism $\varphi_{\mathrm{m}}$ ).

PROOF: See [7; p. 170 and 179] 
REMARKS :

(1) It might be helpful to consider the situation from a geometrical point of view. If $\mathscr{U}$ is the affine plane coordinatized by $S$ then an autotopism is a collineation of $\mathscr{U}$ fixing the coordinate axes with $\varphi_{1}$ describing the action on the $x$-axis, $\varphi_{2}$ the action on the line at infinity, and $\varphi_{3}$ the action on the y-axis. Thus in statement (iii) above we are on the one-hand -when considering $\varphi_{1}$-- looking at the $x$-axis of and on the other -- when considering $\varphi_{2}--$ looking at the line at infinity of $\mathscr{U}$.

(2) There are lemmas corresponding to Lemma 2.1 for both the right nucleus $\mathrm{N}_{\mathrm{r}}$ and the left nucleus $\mathrm{N}_{\ell}$ of $\mathrm{S}$. However, there are some slight differences. The semi-field $\mathrm{S}$ is a right vector space over $\mathrm{N}_{\mathrm{r}}$ and for $\varphi=\left(\varphi_{1}, \varphi_{2}, \varphi_{3}\right) \in G$ the components $\varphi_{2}$ and $\varphi_{3}$ of $\varphi$ are semi-1inear transformations on $\mathrm{S}$ over $\mathrm{N}_{\mathrm{r}}$. The companion automorphism in both cases is $\varphi_{\mathrm{r}}: \mathrm{N}_{\mathrm{r}} \rightarrow \mathrm{N}_{\mathrm{r}}$ given by ${ }^{n} \varphi_{r} \equiv\left(a_{\varphi} b_{\varphi} \mathrm{n}\right) \varphi_{3} \cdot$ Similarly, the semi-field $S$ is a left vector space over $N_{\ell}$ and the components $\varphi_{1}$ and $\varphi_{3}$ are semi-1inear transformations on $S$ over $N_{\ell}$ with companion automorphism $\varphi_{\ell}: N_{\ell} \rightarrow N_{\ell}$, where $n \varphi_{\ell} \equiv\left(\mathrm{na}_{\varphi} \mathrm{b}_{\varphi}\right) \varphi_{3} \cdot \quad$ (See $[7 ; \mathrm{p} .170$ and 179].)

DEFINITION 2.1: Let $\mathrm{S}$ be a finite semi-field of order $\mathrm{p}^{\mathrm{s}}$ with middle nucleus $\mathrm{N}_{\mathrm{m}}$ of order $\mathrm{p}^{\mathrm{t}} \mathrm{m}$ and let $\mathrm{G}$ be the autotopism group of $\mathrm{S}$. The middle linear autotopism group of $\mathrm{S}$ is the subgroup $\underline{L G}$ (S) of $G$ consisting of all autotopisms $\varphi=\left(\varphi_{1}, \varphi_{2}, \varphi_{3}\right)$ with $\varphi_{1}$ and $\varphi_{2}$ linear transformations on $S$ as a vector space over $\mathrm{N}_{\mathrm{m}}$. The middle homomorphisms of $\mathrm{S}$ are the homomorphisms $I_{m j}: L_{m}(S) \rightarrow G L\left(d_{m}, N_{m}\right)$, where $d_{m}=s t_{m}^{-1}$ and $d=1,2$ defined by

$$
\left(\varphi_{1}, \varphi_{2}, \varphi_{3}\right) \Pi_{m j}=\varphi_{j}
$$

LEMMA 2.2: Let $\mathrm{S}$ be a finite semi-field of order $\mathrm{p}^{\mathrm{s}}$, where $\mathrm{p}$ is a prime, let $\mathrm{N}_{\mathrm{m}}$ be the middle nucleus of $\mathrm{S}$ having order $\mathrm{p}^{\mathrm{t}} \mathrm{m}$, and let $\mathrm{G}$ be the autotopism group of $\mathrm{S}$. The following statements hold: 
(i) The middle linear autotopism group $L_{m}(S)$ is the kernel of the homomorphism $\Sigma_{\mathrm{m}}: G \rightarrow \operatorname{Aut}\left(\mathrm{N}_{\mathrm{m}}\right)$ given by $\varphi \Sigma_{\mathrm{m}} \equiv \varphi_{\mathrm{m}}$.

(ii) The integer $\left[G: \mathrm{LG}_{\mathrm{m}}(\mathrm{s})\right]$ divides $\mathrm{t}_{\mathrm{m}}$.

(iii) The kernel of the homomorphism $\pi_{\mathrm{ml}}$ is the group $N_{r}^{*} \equiv\left\{\rho_{n} \mid n \epsilon\right.$ $\left.\mathrm{N}_{\mathrm{r}}-\{0\}\right\}$, where $\rho_{\mathrm{n}}=(1, \rho, \rho)$ with $\rho: \mathrm{s} \rightarrow \mathrm{s}$ given by $\mathrm{x} \rho \equiv \mathrm{xn}$. The group $\mathrm{N}_{\mathrm{r}}{ }^{*}$ is isomorphic to the multiplicative group $\mathrm{N}_{\mathrm{r}}-\{0\}$.

(iv) The kernel of the homomorphism $\pi_{\mathrm{m} 2}$ is the group $\mathrm{N}_{\ell}{ }^{*} \equiv\left\{\lambda_{\mathrm{n}} \mid\right.$. $\left.\mathrm{n} \in \mathrm{N}_{\ell}-\{0\}\right\}$ where $\lambda_{\mathrm{n}}=(\lambda, 1, \lambda)$ with $\lambda: \mathrm{s} \rightarrow \mathrm{s}$ given by $\mathbf{x} \lambda \equiv \mathbf{n} \mathbf{x}$. The group $\mathrm{N}_{\ell}{ }^{*}$ is isomorphic to the multiplicative group $\mathrm{N}_{\ell}-\{0\}$.

PROOF: Statement (i) is obvious and statement (ii) follows from (i). For statement (iii) note first that $\mathrm{N}_{\mathrm{r}}{ }^{*} \leq$ ker $\Pi_{\mathrm{ml}}$. For given $\dot{\rho}_{\mathrm{n}}=(1, \rho, \rho)$ we have $a_{\rho n}=1$ and $b_{\rho n}=n^{-1}$; thus for $x \in N_{m}$ we have $x\left(\rho_{n}\right)_{m}=\left(x^{-1}\right) \rho=\left(x n^{-1}\right) n=x$. Assume now that $\varphi=\left(\varphi_{1}, \varphi_{2}, \varphi_{3}\right) \in \operatorname{ker} \pi_{\mathrm{m} 1}$. Then $\varphi_{1}=1$; hence for all $\mathrm{x}, \mathrm{y} \in \mathrm{s}$ we have $x\left(y \varphi_{2}\right)=(x y) \varphi_{3}$. Letting $x=1$ gives $\varphi_{2}=\varphi_{3}$. If $n \equiv 1 \varphi_{2}$ then $x \varphi_{3}=x n$ for all $x \in S$. Thus $x(y n)=(x y) n$ for all $x, y \in S$. Hence $n \in N_{r}-\{0\}$ and $\varphi=(1, \rho, \rho)$ with $\rho: x \rightarrow x$ for all $x \in S$. This proves (iii). The proof of statement (iv) is similar.

REMARKS :

(1) Analogous to the group $L G_{m}(S)$ we have a right linear autotopism group $\underline{L G}_{r}(\underline{S})$ - consisting of all $\varphi=\left(\varphi_{1}, \varphi_{2}, \varphi_{3}\right)$ with $\varphi_{2}$ and $\varphi_{3}$ linear transformations over $\mathrm{N}_{\mathrm{r}}$ - and a left $\underline{\text { linear }} \underline{\text { autotopism }} \underline{\text { group }} \underline{\mathrm{LG}} \underline{l}_{l}(\mathrm{~S})$ - consisting of all $\dot{\varphi}=\left(\varphi_{1}, \varphi_{2}, \varphi_{3}\right)$ with $\varphi_{1}$ and $\varphi_{2}$ linear transformations over $N_{\ell}$. In turn these groups have associated with them mappings $\Pi_{r i}: L G_{r}(S) \rightarrow G L\left(d_{r}, N_{r}\right)$ and $\Pi_{\ell i}: L G_{\ell}(s) \rightarrow G L\left(d_{\ell}, N_{\ell}\right)-$ here $d_{\alpha}$ is the dimension of $s$ over $N_{\alpha}$ - where

$$
\begin{array}{ll}
\left(\varphi_{1}, \varphi_{2}, \varphi_{3}\right) \Pi_{r i} \equiv \varphi_{i} & i=2,3 \\
\left(\varphi_{1}, \varphi_{2}, \varphi_{3}\right) \Pi_{\ell i} & \varphi_{i} \quad i=1,3
\end{array}
$$


Results similar to the statements of Lemma 2.2 are true. In particular, ker $\Pi_{r 1}=N_{\ell}^{*}$, ker $\Pi_{\ell 1}=N_{r}^{*}$, and ker $\Pi_{r 2}=\operatorname{ker} \Pi_{\ell 2}=N_{m}^{*} \equiv\left\{\mu_{n} \mid n \in N_{m}-\{0\}\right\}$ where $\mu_{n}=\left(\mu_{1}, \mu_{2}, 1\right)$ is given by

$$
\mathrm{x} \mu_{1} \equiv \mathrm{xn}, \quad \mathrm{x} \mu_{2} \equiv \mathrm{n}^{-1} \mathrm{x} .
$$

for $x \in S$. We can then define

$$
\underline{L G(S)} \equiv \prod_{\alpha} L G(S)
$$

where $\alpha$ runs over $\{r, m, l\}$. This is called the linear autotopism group of $s$. The mappings $\Pi_{\alpha i}$ could then be restricted to the group LG(S).

(2) In the remainder of this article, whenever we consider the group $\mathrm{LG}_{\mathrm{m}}(\mathrm{S})$ we will frequently restrict ourselves to one of the groups $G_{m i}=\left\{\varphi_{1} \mid \varphi=\left(\varphi_{1}, \varphi_{2}, \varphi_{3}\right)\right.$ $\left.\in L G_{m}(S)\right\}$ for $i=1$ or 2 . These groups are really homomorphic images of $L G_{m}(S)$ and the purpose of the above lemma is to make this clear. Similar statements hold for the groups $L G_{r}(S)$ and $L G_{\ell}(S)$.

LEMMA 2.3: Let $\mathrm{S}$ be a finite semi-field of order $\mathrm{p}^{\mathrm{r}}$, where $\mathrm{p}$ is a prime, and let $G$ be the autotopism group of $S$. The group $G$ is solvable if and only if the subgroup $\mathrm{LG}_{\mathrm{m}}(\mathrm{S})$ is solvable.

PROOF: This follows from the fact the $G / L G_{m}(S)$ is a subgroup of $\operatorname{Aut}\left(N_{m}\right)$. REMARK: In Lemma 2.3 , the group $L G_{m}(S)$ can be replaced with any of the groups $L G_{r}(S)$, and $L G_{\ell}(S)$. The last group is permissible because its definition implies that the factor group $G / L G(S)$ is a subgroup of the direct product $\gg G_{\alpha} / L G_{\alpha}(S)$, where $\alpha=r, m, l$. (See $[8 ; 1.9 .6]$.).

DEFINITION 2.2: Let $\mathrm{S}$ be a finite semi-field of order $\mathrm{p}$, where $\mathrm{p}$ is a prime. For $i=1,2$ we define $D_{m i}$ to be the group of all $\varphi=\left(\varphi_{1}, \varphi_{2}, \varphi_{3}\right)$ in $L G_{m}(S)$ with det $\varphi_{i}=1$, and we define $\underline{D}_{m}=D_{m}(\underline{S})$ to be the group $D_{m 1} \cap D_{m 2}$. The group $\mathrm{D}_{\mathrm{m}}$ is the middle determinant group of $\mathrm{s}$.

LEMMA 2.4: Let $\mathrm{S}$ be a finite semi-field of order $\mathrm{p}^{\mathrm{s}}$ with $\mathrm{p}$ a prime and let $\mathrm{G}$ be the autotopism group of $\mathrm{S}$. The following statements hold: 
(i) The subgroups $D_{m 1}, D_{m 2}$, and $D_{m}$ are normal in $L G_{m}(S)$.

(ii) The group $G$ is solvable if and only if one (and hence all) of the groups $\mathrm{D}_{\mathrm{m} 1}, \mathrm{D}_{\mathrm{m} 2}$, and $\mathrm{D}_{\mathrm{m}}$ is solvable.

(iii) The integers $\left|\mathrm{LG}_{\mathrm{m}}(\mathrm{S})\right|\left|\mathrm{D}_{\mathrm{ml}}\right|^{-1}$ and $\left|\mathrm{LG}_{\mathrm{m}}(\mathrm{S})\right|\left|\mathrm{D}_{\mathrm{m} 2}\right|^{-1}$ divide $\mathrm{p}^{\mathrm{t} m}-\mathrm{L}$.

PROOF: Statements (i) and (ii) follow from the fact that $\mathrm{D}_{\mathrm{mi}}$ is the kernel of the homomorphism $\bar{\Pi}_{m i}: L G_{m}(S) \rightarrow N_{m}-\{0\}$ given by $\left(\varphi_{1}, \varphi_{2}, \varphi_{3}\right) \bar{\Pi}_{m i}$ $=\operatorname{det} \varphi_{i}$. The proof of statement (iii) is similar to the proof of Lemma 2.3.

REMARK: We can also define subgroups $D_{r 2}, D_{r 3}, D_{r}$ of $L G_{r}(S)$ and $\mathrm{D}_{\ell 1}, \mathrm{D}_{\ell 3}, \mathrm{D}_{\ell}$ of $\mathrm{LG}_{\ell}(\mathrm{S})$. Lemma similar to Lemma 2.4 can then be proven. Thus for each such group $D_{\alpha j}$, we have

$$
|G|=u_{\alpha} v_{\alpha j}\left|D_{\alpha j}\right|
$$

with $u_{\alpha} \mid t_{\alpha}$ and $v_{\alpha j} \mid\left(p^{t \alpha}-1\right)$. Also, the group $G$ is solvable if and only if one (and hence all) of the $D_{\alpha j}$ is solvable.

DEFINITION 2.3: Let $\mathrm{s}$ be a finite semi-field of order $\mathrm{p}^{\mathrm{s}}$ with autotopism group G. The determinant group of $S$ is the subgroup $\underline{D}=\underline{D(S)}=D_{r} \cap D_{m} \cap D_{\ell}$ of $G$.

We close this section with a lemma that plays a role similar to the role of Lemma 2.3 .

LEMMA 2.5: Let $\mathrm{S}$ be a finite semi-field of order $\mathrm{p}^{\mathrm{s}}$ with $\mathrm{p}$ a prime and let its middle nucleus $\mathrm{N}_{\mathrm{m}}$ have order $\mathrm{p}^{\mathrm{t}} \mathrm{m}$. For $\mathrm{i}=1$ or 2 the homomorphism $\Pi_{m i}$ induces by restriction a homomorphism $\pi_{m i}^{*}: D_{m i} \rightarrow S L\left(d_{m}, N_{m}\right)$, where $\mathrm{d}_{\mathrm{m}}=\mathrm{st}_{\mathrm{m}}^{-1}$, and ker $\Pi_{\mathrm{ml}}^{*}=\mathrm{D}_{\mathrm{m} 1} \cap \mathrm{N}_{\mathrm{r}}^{*}$ and ker $\Pi_{\mathrm{m} 2}^{*}=\mathrm{D}_{\mathrm{m} 2} \cap \mathrm{N}_{\ell}^{*}$.

\section{THE DIMENSION 2 CASE.}

In this section we restrict ourselves to semi-fields $S$ which have dimension 2 over one of their nuclei. Without loss of generality, we shall assume the nucleus is $\mathrm{N}_{\mathrm{m}}$. (By this we mean that similar arguments apply for either of the other two nuclei. Thus, in the notation of the previous section, we have 


$$
\begin{aligned}
& d_{m}=\operatorname{dim}_{N_{m}} s=s t_{m}^{-1}=2 \\
& s=2 t_{m} .
\end{aligned}
$$

Consider first the group $\mathrm{LG}_{\mathrm{m}}(\mathrm{S})$. The following result is important in the analysis of the structure of $L G_{m}(S)$ and the subgroups $D_{m j}$.

THEOREM 3.1: Let $\mathrm{S}$ be a finite semi-field of order $\mathrm{p}^{\mathrm{s}}$ where $\mathrm{p}$ is a prime and assume that $S$ has dimension 2 over its middle nucleus $N_{m}$. If $p>2$ then the group $\mathrm{LG}_{\mathrm{m}}(\mathrm{S})$ contains no elements of order $\mathrm{p}$.

PROOF: Assume $\varphi=\left(\varphi_{1}, \varphi_{2}, \varphi_{3}\right)$ is an element of order $p$ in $\mathrm{LG}_{\mathrm{m}}(\mathrm{S})$. Consider the projective plane $P(S)$ coordinatized by $S$. Then $\varphi$ induces a collineation $\bar{\varphi}$ on $P(S)$ fixing the points $(0,0),(0)$, and $(\infty)$. (See Remark (1) after Lemma 2.1.) Because $\bar{\varphi}$ has order p, it fixes other points in each of the three lines determined by these points, hence it fixes a subplane $P_{0}$ pointwise. Consider the action of $\bar{\varphi}$ on the line $(0,0)(0)$; this is given by

$$
(\mathrm{x}, 0) \bar{\varphi}=\left(\mathrm{x} \varphi_{1}, 0\right) \text {. }
$$

Since $\bar{\varphi}$ cannot be an elation, we must have $\varphi_{1} \neq 1$. Thus, $\varphi_{1}$ is a linear transformation of order $p$; that is, $\varphi_{1} \in G L\left(2, N_{m}\right)$. An element of order $p$ in $\operatorname{GL}\left(2, N_{m}\right)$ fixes exactly $\left|N_{m}\right|=p^{t_{m}}$ points. It follows that $\bar{\varphi}$ fixes pointwise a subplane $p_{0}$ of order $p^{t}$ and thus $\bar{\varphi}$ is a Baer collineation. By Foulser [1; Theorem 4.3], this gives a collineation. Hence the theorem holds.

For later reference, we state a result, due to Ganley [2], for the case $\mathrm{p}=2$.

THEOREM 3.2: GANLEY: Let $\mathrm{S}$ be a finite semi-field of order $2^{\mathrm{S}}$. If $\mathrm{S}$ has dimension 2 over one of its nuclei then its autotopism group is solvable.

Consider now the subgroup $D_{m l}$ of $L_{m}(S)$. Since $D_{m l}$ is the kernel of the homomorphism $\bar{\Pi}_{\mathrm{ml}}: \mathrm{LG}_{\mathrm{m}}(\mathrm{S}) \rightarrow \mathrm{GL}\left(\left(2, \mathrm{~N}_{\mathrm{m}}\right) / \mathrm{SL}\left(2, \mathrm{~N}_{\mathrm{m}}\right)\right.$, the homomorphism $\pi_{\mathrm{ml}}$ maps $\mathrm{D}_{\mathrm{ml}}$ onto a subgroup $\mathrm{D}_{\mathrm{ml}}^{*}$ of $\mathrm{SL}\left(2, \mathrm{~N}_{\mathrm{m}}\right)$ with kernel $\mathrm{D}_{\mathrm{m} 1} \cap \mathrm{N}_{\mathrm{r}}^{*}$. (See Lemma 2.4.) 
We will use this fact repeatedly.

THEOREM 3.3: Let $\mathrm{S}$ be a semi-field of order $\mathrm{p}^{\mathrm{s}}$, where $\mathrm{p}$ is a prime, and having dimension 2 over its middle nucleus $\mathrm{N}_{\mathrm{m}}$, let $\mathrm{G}$ be its autotopism group, let $\mathrm{D}_{\mathrm{ml}}^{*}=\mathrm{D}_{\mathrm{ml}} /\left(\mathrm{D}_{\mathrm{ml}} \cap \mathrm{N}_{\mathrm{r}}^{*}\right)$, let $\left|\mathrm{N}_{\mathrm{m}}\right|=\mathrm{p}^{\mathrm{t}} \mathrm{m}$, and let $\left|\mathrm{N}_{\mathrm{r}}\right|=\mathrm{p}^{\mathrm{t} r}$. One and only one of the following statements holds:

(i) The autotopism group $G$ is non-solvable with $\mathrm{D}_{\mathrm{ml}}^{*}=\mathrm{SL}(2,5)$ and $|G|$ divides $t_{m}\left(p^{t} m-1\right)\left(p^{t} r-1\right) \cdot 120=60 s\left(p^{\frac{1}{2} s}-1\right)\left(p^{t} r-1\right)$.

Furthermore, $\mathrm{p} \geq 7$ and $\mathrm{p}^{\mathrm{s}}-1 \equiv 0(\bmod 5)$.

(ii) The autotopism group $G$ is solvable with $\mathrm{D}_{\mathrm{ml}}^{*}$ a solvable subgroup of $\operatorname{SL}\left(2, \mathrm{~N}_{\mathrm{m}}\right)$ such that $\left(\mathrm{p},\left|\mathrm{D}_{\mathrm{ml}}^{*}\right|\right)=1$ and $|\mathrm{G}|$ divides $t_{m}\left(p^{t} m-1\right)\left(p^{t} r-1\right) k=\frac{1}{2} s\left(p^{\frac{1}{2} s}-1\right)\left(p^{t} r-1\right)$ where either $k \mid 2\left(p^{\frac{1}{2} s} \pm 1\right), k=24$, or $k=48$.

PROOF: By the Remark after Lemma 2.3, the integer $|G|\left|D_{m l}\right|^{-1}$ divides $t_{m}\left(p^{t} m-1\right)$. Consider the group $d_{m l}$. The group $D_{m l}^{*}=D_{m l} /\left(D_{m l} \cap N_{r}^{*}\right)$ is a subgroup of $\mathrm{SL}\left(2, \mathrm{~N}_{\mathrm{m}}\right)=\mathrm{SL}\left(2, \mathrm{p}^{\mathrm{t}} \mathrm{m}\right)$ containing no p-elements (Theorem 3.1). If $\mathrm{G}$ is non-solvable, then $\mathrm{D}_{\mathrm{ml}}$ is non-solvable and so is $\mathrm{D}_{\mathrm{ml}}^{*}$. It follows that $\mathrm{D}_{\mathrm{ml}}^{*}=\mathrm{SL}(2,5)$. (See Huppert [8; Hauptsatz II.8.27].)

If $\mathrm{G}$ is solvable, then $\mathrm{D}_{\mathrm{ml}}^{*}$ must be a solvable subgroup of $\mathrm{sL}\left(2, \mathrm{p}^{\mathrm{t}} \mathrm{m}\right)$ having no p-elements; then $D_{m l}^{*}$ must either have order dividing $2\left(p^{t} m \pm 1\right)$ or its image in $\operatorname{PSL}\left(2, \mathrm{p}^{\mathrm{t}} \mathrm{m}\right.$ ) is either $\mathrm{A}_{4}$ or $\mathrm{S}_{4}$. (Again, see Hauptsatz II.8.27 of [8].

REMARK: Note that Theorem 3.3 has analogies with $\mathrm{N}_{\mathrm{m}}$ and $\mathrm{D}_{\mathrm{ml}}$ replaced by $\mathrm{N}_{\alpha}$ and $\mathrm{D}_{\alpha \mathrm{j}}$, where $\alpha \in\{\mathrm{r}, \mathrm{m}, \mathrm{l}\}$ and $\mathrm{j} \in\{1,2,3\}$.

We investigate further statement (i) above by means of a sequence of lemmas.

LEMMA 3.1: Let $\mathrm{s}$ be a semi-field of order $\mathrm{p}^{\mathrm{s}}$, where $\mathrm{p}$ is an odd prime and having dimension 2 over its middle nucleus $\mathrm{N}_{m}$, let $G$ be its autotopism group, and let $D_{m l}^{*}=D_{m l} /\left(D_{m} \cap N_{r}^{*}\right)$. If $G$ is non-solvable, then the following statements hold:

$$
\text { (i) } \mathrm{D}_{\mathrm{ml}}^{*}=\mathrm{SL}(2,5)
$$


(ii) $\left|\mathrm{D}_{\mathrm{ml}} \cap \mathrm{N}_{\mathrm{m}}^{*}\right|=2=\left|\mathrm{D}_{\mathrm{ml}} \cap \mathrm{N}_{\ell}^{*}\right|$

PROOF: Statement (i) follows from statement (i) of Theorem 3.3. For statement (ii) note that $E=D_{m l} \cap N_{m}^{*}$ and $F=D_{m l} \cap N_{l}^{*}$ are normal cyclic subgroups of $\mathrm{D}_{\mathrm{ml}}$. Since $\mathrm{N}_{\mathrm{m}}^{*} \cap \mathrm{N}_{\mathrm{r}}^{*}=\mathrm{N}_{\mathrm{m}}^{*} \cap \mathrm{N}^{*}=1$, it follows that $\mathrm{E}$ and $\mathrm{F}$ yield cyclic normal subgroups $E^{*}$ and $F^{*}$ of $D_{m l}^{*}$ with $E^{*} \simeq E$ and $F^{*} \simeq F$. Now $D_{m l}^{*}$ has exactly two such subgroups; namely, 1 and its center $Z$ of order 2 . Since the autotopisms $\sigma_{\mathrm{m}}=(\varepsilon, \varepsilon, 1)$ and $\sigma_{\ell}=(\varepsilon, 1, \varepsilon)$, where $\varepsilon: \mathrm{x} \rightarrow(-1) \mathrm{x}=\mathrm{x}(-1)=-\mathrm{x}$, are in $E$ and $F$, respectively, we have $E^{*}=Z=F^{*}$.

Under the hypothesis of Lemma 3.1, consider the group $\mathrm{D}_{\mathrm{m} 2}^{\star \star}=\mathrm{D}_{\mathrm{m} 2} /\left(\mathrm{D}_{\mathrm{m} 2} \cap \mathrm{N}_{\ell}^{*}\right)$. By arguments similar to those given in the first paragraph of the proof of Theorem 3.3 and in the proof of Lemma 3.1 , we have the following Lemma.

LEMMA 3.2: Under the hypothesis of Lemma 3.1 the following statements hold for the group $\mathrm{D}_{\mathrm{m} 2}^{*}=\mathrm{D}_{\mathrm{m} 2} /\left(\mathrm{D}_{\mathrm{m} 2} \cap \mathrm{N}_{\ell}^{*}\right)$ :

$$
\begin{aligned}
& \text { (i) } \mathrm{D}_{\mathrm{m} 2}^{* *}=\mathrm{SL}(2,5) \\
& \text { (ii) }\left|\mathrm{D}_{\mathrm{m} 2} \cap \mathrm{N}_{\mathrm{m}}^{*}\right|=\left|\mathrm{D}_{\mathrm{m} 2} \cap \mathrm{N}_{\mathrm{r}}^{*}\right|=2
\end{aligned}
$$

Consider now the group $\mathrm{D}_{\mathrm{m}}=\mathrm{D}_{\mathrm{ml}} \cap \mathrm{D}_{\mathrm{m} 2}$. Under the hypothesis of Lemma 3.1 , the group $D_{m}$ is non-solvable (Lemma 2.4). Hence $D_{m}$ yields a non-solvable normal subgroup $\mathrm{D}_{\mathrm{m}}^{*}$ of $\mathrm{D}_{\mathrm{ml}}^{*}=\mathrm{SL}(2,5)$. It follows that $\mathrm{D}_{\mathrm{m}}^{*}=\mathrm{D}_{\mathrm{ml}}^{*}$. Since $\left|\mathrm{D}_{\mathrm{ml}} \cap \mathrm{N}_{\mathrm{r}}^{*}\right|=2$, we have $\mathrm{D}_{\mathrm{m}}=\mathrm{D}_{\mathrm{ml}}$. Similarly, $\mathrm{D}_{\mathrm{m}}=\mathrm{D}_{\mathrm{m} 2}$. Thus $\mathrm{D}_{\mathrm{m}}=\mathrm{D}_{\mathrm{m} 1}=\mathrm{D}_{\mathrm{m} 2}$ and in the last two lemmas $\mathrm{D}_{\mathrm{m}}$ can replace $\mathrm{D}_{\mathrm{m} 1}$ and $\mathrm{D}_{\mathrm{m} 2}$.

Let $\sigma=\left(\sigma_{1}, \sigma_{2}, \sigma_{3}\right)$ be an involution in $\mathrm{D}_{\mathrm{m}}$. If $\sigma$ is a Baer involution (that is, if each $\sigma_{i}$ fixes pointwise a subspace $S_{i}$ of $S$ having dimension $\frac{1}{2} s$ over $\left.G F(p)\right)$, then its image $\sigma^{*}$ in $\mathrm{D}_{\mathrm{m}}^{*}=\mathrm{D}_{\mathrm{ml}}^{*}$ must be a non-trivial involution in $\mathrm{D}_{\mathrm{m}}^{*}=\mathrm{SL}(2,5)$ fixing a non-trivial subspace pointwise. But $\mathrm{D}_{\mathrm{m}}^{*}=\mathrm{SL}(2,5)$ has a unique involution and, since $\sigma_{\mathrm{m}}=(\varepsilon, \varepsilon, 1)$ is $\mathrm{D}_{\mathrm{m}}^{*}$, the unique involution of $\mathrm{D}_{\mathrm{m}}^{*}$ is $\varepsilon$ which has no fixed points. Thus we have a contradiction and hence 
$\mathrm{D}_{\mathrm{m}}$ has no Baer involution.

If $\sigma=\left(\sigma_{1}, \sigma_{2}, \sigma_{3}\right)$ is an involution in $\mathrm{D}_{\mathrm{m}}$, then each $\sigma_{1}$ is either the identity 1 or the mapping $\varepsilon$. Since at most one $\sigma_{i}$ can be 1 , the group $D_{m}$ has exactly three involutions - namely,

$$
\sigma_{\mathrm{r}} \equiv(1, \varepsilon, \varepsilon), \quad \sigma_{\mathrm{m}} \equiv(\varepsilon, \varepsilon, 1), \quad \text { and } \quad \sigma_{\ell} \equiv(\varepsilon, 1, \varepsilon) .
$$

Note that $\mathrm{D}_{\mathrm{m}} \cap \mathrm{N}_{\alpha}^{*}=\left\langle\sigma_{\alpha}\right\rangle$ for $\alpha=\mathrm{r}, \mathrm{m}, \mathrm{l}$. Furthermore, direct calculation shows that for all $\tau \in D_{m}$, we have $\sigma_{\alpha} \tau=\tau \sigma_{\alpha}$ for $\alpha=r, m, l$. Hence $z=\left\{1, \sigma_{r}, \sigma_{m}, \sigma_{l}\right\}$ is a subgroup of $\mathrm{Z}\left(\mathrm{D}_{\mathrm{m}}\right)$. Since $\mathrm{D}_{\mathrm{m}}^{*}=\mathrm{D}_{\mathrm{m}} /<\sigma_{\mathrm{r}}>$ has a center of order 2 , it follows that $Z\left(D_{m}\right)=Z$.

We have proven the following lemma.

LEMMA 3.3: Under the hypothesis of Lemma 3.1, the following statements hold:

(i) $\mathrm{D}_{\mathrm{m}}=\mathrm{D}_{\mathrm{ml}}=\mathrm{D}_{\mathrm{m} 2}$

(ii) $\left|D_{m}\right|=240$

(iii) $\mathrm{D}_{\mathrm{m}}$ contains exactly three involutions; namely $\sigma_{\mathrm{r}}=(1, \varepsilon, \varepsilon), \sigma_{\mathrm{m}}=(\varepsilon, \varepsilon, 1)$, and $\sigma_{\ell}=(\varepsilon, 1, \varepsilon)$ where $\varepsilon: \mathrm{S} \rightarrow \mathrm{S}$ is given by $\varepsilon: \mathrm{x} \rightarrow(-1) \mathrm{x}=\mathrm{x}(-1)=-\mathrm{x}$.

(iv) $\mathrm{Z}\left(\mathrm{D}_{\mathrm{m}}\right)=\left\{1, \sigma_{\mathrm{r}}, \sigma_{\mathrm{m}}, \sigma_{\ell}\right\}$

(v) $\mathrm{D}_{\mathrm{m}} \cap \mathrm{N}_{\alpha}^{*}=\left\langle\sigma_{\alpha}>\right.$ for al1 $\sigma \in\{\mathrm{r}, \mathrm{m}, \mathrm{l}\}$

We turn to investigating the Sylow 2-subgroups of $\mathrm{D}_{\mathrm{m}}$. Assume $\tau \in \mathrm{D}_{\mathrm{m}}$ is an element of order 8. Then $\tau^{4}$ is an involution in $D_{m}$ and hence $\tau^{4}=\sigma_{\alpha}$ for some $\alpha \in\{r, m, l\}$. If $\alpha \neq r$ then $\tau$ induces an element $\tau^{*}$ of order 8 in $\mathrm{D}_{\mathrm{m}}^{*}=\mathrm{D}_{\mathrm{m}} /<\sigma_{\ell}>=\mathrm{SL}(2,5)$. If $\alpha=\mathrm{r}$ then $\tau$ induces an element $\tau^{* *}$ of order 8 in $\mathrm{D}_{\mathrm{m}}^{* *}=\mathrm{D}_{\mathrm{m}} /<\sigma_{\ell}>=\mathrm{SL}(2,5)$. Since $\mathrm{SL}(2,5)$ has no element of order 8 , in both cases we have a contradiction. Thus, $D_{m}$ has no elements of order 8 .

Let $\mathrm{T}$ be a Sylow 2-subgroup of $\mathrm{D}_{\mathrm{m}}$. The group $\mathrm{T}$ contains the center $Z=Z\left(D_{m}\right)$ of $D_{m}$. Furthermore, the image $T^{*}$ of $T$ in the factor group 
$\mathrm{D}_{\mathrm{m}}^{*}=\mathrm{SL}(2,5)$ is a quaternion group of order 8 . The normalizer of $\mathrm{T}^{*}$ in $\mathrm{D}^{*}$ contains an element $\tau^{*}$ of order 3 that does not centralize $T^{*}$. (This follows from Hauptsatz II.8.27 of [8] as applied to PSL $(2,5)$.) If $\tau \in D_{m}$ is a preimage in $D_{m}$ of $\tau^{*}$ with $|\tau|=3$, then $\tau$ normalizes $T$ but does not centralize $T$. Hence the group $\mathrm{T}$ has a non-trivial automorphism of order 3 .

Thus, $\mathrm{T}$ is a group of order 16 having exactly 3 involutions, no elements of order 8, and an automorphism of order 3. Consulting the list in [3] of the 14 groups of order 16, we see that there is exactly one such group-namely, $\mathrm{Q} \otimes \mathrm{z}_{2}$ - having these three properties.

Consider the group $D_{m} \cap D_{r}$. Since $G$ is non-solvable and $G /\left(D_{m} \cap D_{r}\right)$ is isomorphic to a subgroup of $G / D_{m} \otimes G / D_{r}$, by Lemma 2.4 and the Remark following, we have $D_{m} \cap D_{r}$ is non-solvable. Hence its image $\left(D_{m} \cap D_{r}\right)^{*}$ in $D_{m}^{*}$ is a nonsolvable normal subgroup of $\mathrm{D}_{\mathrm{m}}^{*}=\mathrm{SL}(2,5)$. It follows that $\left(\mathrm{D}_{\mathrm{m}} \cap \mathrm{D}_{\mathrm{r}}\right)^{*}=\mathrm{D}_{\mathrm{m}}^{*}$. Since $D_{m}^{*}=D_{m} /\left\langle\sigma_{r}\right\rangle$ and $\left\langle\sigma_{r}\right\rangle \leq D_{m} \cap D_{r}$, we have that $D_{m} \cap D_{r}=D_{m}$ or $D_{m} \leq D_{r}$. A similar proof shows that $D_{m} \leq D$. Thus, $D(S)=D_{m}$. (See Definition 2.3.)

Now the group $T=Q \otimes z_{2}$ has the property that for one of its involutions in this case $\sigma_{m}$-the factor group $T /\left\langle\sigma_{m}\right\rangle$ is elementary abelian. Since $\mathrm{D}_{\mathrm{m}} /<\sigma_{\mathrm{m}}>$ is a homomorphic pre-image of $\mathrm{D}_{\mathrm{m}} / Z\left(\mathrm{D}_{\mathrm{m}}\right)=\operatorname{PSL}(2,5)$, it follows that $D_{m} /<\sigma_{m}>=\operatorname{PSL}(2,5) \otimes Z_{2}$.

We have proven the following lemma.

LEMMA 3.4: Under the hypothesis of Lemma 3.1, the following statements hold:

(i) The Sylow 2-subgroups of $D_{m}$ are isomorphic to $Q \otimes z_{2}$

(ii) $\mathrm{D}(\mathrm{S})=\mathrm{D}_{\mathrm{m}}$

(iii) $\mathrm{D}_{\mathrm{m}} /<\sigma_{\mathrm{m}}>=\operatorname{PSL}(2,5) \otimes \mathrm{Z}_{2}$

It follows from the various Remarks made in Section 2 that the proof of Lemmas 3.1 - 3.4 can be applied when $N_{m}$ is replaced by $N_{\alpha}$, and thus $D_{m}$ by $D_{\alpha}$, for $\alpha \in\{r, m, l\}$. Thus Lemmas 3.1 and 3.4 can be combined to give the 


\section{following theorem.}

THEOREM 3.4: Let $\mathrm{S}$ be a finite semi-field of order $\mathrm{p}^{\mathrm{s}}$, where $\mathrm{p}$ is an odd prime, and dimension 2 over one of its nuclei $\mathrm{N}_{\alpha}(\alpha \in\{r, m, l\})$. Let $G$ be the autotopism group of $\mathrm{S}$, let $\mathrm{D}_{\alpha}=\mathrm{D}_{\alpha}(\mathrm{S})$ be the determinant group of $\mathrm{S}$ associated with $N_{\alpha}$, and let $D(S) \equiv D_{r} \cap D_{m} \cap D_{\ell}$. If $G$ is nonsolvable then the following statements hold:

(i) $\left|D_{\alpha}\right|=240$

(ii) $\mathrm{D}_{\alpha}$ has exactly three involutions; namely, $\sigma_{r}, \sigma_{m}$, and $\sigma_{\ell}$. (See (3.1))

$$
\begin{aligned}
& \text { (iii) } \mathrm{Z}\left(\mathrm{D}_{\alpha}\right)=\left\{1, \sigma_{\mathrm{r}}, \sigma_{\mathrm{m}}, \sigma_{\ell}\right\} \\
& \text { (iv) } \mathrm{D}_{\alpha} \cap \mathrm{N}_{\beta}^{*}=\left\langle\sigma_{\beta}\right\rangle \text { for all } \beta \in\{\mathrm{r}, \mathrm{m}, \mathrm{l}\} \\
& \text { (v) } \mathrm{D}(\mathrm{S})=\mathrm{D}_{\alpha} \\
& \text { (vi) } \mathrm{D}_{\alpha} /<\sigma_{\beta}>=\operatorname{sL}(2,5) \text { for } \beta \in\{\mathrm{r}, \mathrm{m}, l\}-\{\alpha\} \\
& \text { (vii) } \mathrm{D}_{\alpha} /<\sigma_{\alpha}>=\operatorname{PSL}(2,5) \otimes \mathrm{z}_{2}
\end{aligned}
$$

We close this section with an interesting relationship among the nuclei when the group $\operatorname{SL}(2,5)$ occurs.

COROLLARY 3.4.1: Under the hypothesis of Theorem 3.4, if $\beta, \gamma \epsilon$ $\{\mathbf{r}, \mathbf{m}, \ell\}-\{\alpha\}$ then $\mathrm{N}_{\beta}=\mathrm{N}_{\gamma} \subseteq \mathrm{N}_{\alpha}$.

PROOF: We prove this theorem for $\alpha=m$. Let $n \in N_{r}-\{0\}$ and consider the associated autotopism $\rho_{\mathrm{n}}=(1, \rho, \rho)$, where $\rho: \mathrm{x} \rightarrow \mathrm{xn}$. Consider $\mathrm{D}_{\mathrm{m}} /\left\langle\sigma_{\ell}\right\rangle$ and $\mathrm{G} / \mathrm{N}_{\ell}^{*}$. Such $\left\langle\sigma_{\ell}\right\rangle=\mathrm{D}_{\mathrm{m}} \cap \mathrm{N}_{\ell}^{*}$, we have $\mathrm{D}_{\mathrm{m}} /\left\langle\sigma_{\ell}\right\rangle=\operatorname{SL}(2,5)$ normal in $\mathrm{G} / \mathrm{N}^{*}$ which in turn is a subgroup of $\mathrm{GL}\left(2, \mathrm{~N}_{\mathrm{m}}\right)$. If $\sigma=\left(\sigma_{1}, \sigma_{2}, \sigma_{3}\right)$ is in $\mathrm{D}_{\mathrm{m}}$ then its image in $\mathrm{D}_{\mathrm{m}} /\left\langle\sigma_{\ell}\right\rangle$ is just $\sigma_{2}$. Furthermore, the image of $\rho_{\mathrm{n}}$ in $\mathrm{G} / \mathrm{N}^{*}$ is just $\rho$. Since $\tau_{2}$ is a linear transformation over $N_{r}$ (Remark (2) after Lemma 2.1 and the Remark after Lemma 2.4), we must have $\rho \sigma_{2}=\sigma_{2} \rho$. Thus $\rho$ is in the centralizer of $\operatorname{SL}(2,5)$ in $\mathrm{GL}\left(2, \mathrm{~N}_{\mathrm{m}}\right)$. Since the centralizer of $\mathrm{SL}(2,5)$ in $\mathrm{GL}\left(2, \mathrm{~N}_{\mathrm{m}}\right)$ is the center of GL $\left(2, N_{m}\right)$ - See Huppert [8; Sec. II.7 and II.8] - the mapping $\rho$ is 
in the center of GL $\left(2, N_{m}\right)$. Thus, $\rho: x \rightarrow \bar{n} x$ with $\bar{n} \in N_{m}$. Since $1 \rho=n$ we have $\overline{\mathbf{n}}=\mathrm{n}$. Therefore, $\mathrm{N}_{\mathrm{r}} \subseteq \mathrm{N}_{\mathrm{m}}$. Note also that this implies for all $\mathrm{n} \in \mathrm{N}_{\mathrm{r}}$ we have $\mathrm{nx}=\mathrm{xn}$ for all $\mathrm{x} \in \mathrm{s}$. Thus, if $\mathrm{n} \in \mathrm{N}_{\mathrm{r}}$ and $\mathrm{x}, \mathrm{y} \in \mathrm{s}$, then

$$
\mathrm{n}(\mathrm{xy})=(\mathrm{xy}) \mathrm{n}=\mathrm{x}(\mathrm{yn})=\mathrm{x}(\mathrm{ny})=(\mathrm{xn}) \mathrm{y}=(\mathrm{nx}) \mathrm{y}
$$

This says that $\mathrm{n} \in \mathrm{N}_{\ell}$. Hence $\mathrm{N}_{\mathrm{r}} \subseteq \mathrm{N}$ also.

To show that $\mathrm{N}_{\ell} \subseteq \mathrm{N}_{\mathrm{r}} \subseteq \mathrm{N}_{\mathrm{m}}$, consider the mapping $\lambda_{\mathrm{n}}$, where $\mathrm{n} \in \mathrm{N}$

(Lemma 2.2(iv)), and the groups $\mathrm{D}_{\mathrm{m}} /\left\langle\sigma_{\mathrm{r}}\right\rangle$ and $\mathrm{G} / \mathrm{N}_{\mathrm{r}}^{*}$. Proceeding as in the preceding paragraph, we obtain the desired result.

REMARK: Note that we have actually shown in the proof of Corollary 3.4.1 that $\mathrm{N}_{\sigma}=\mathrm{N}_{\beta}$ is the center of $\mathrm{S}$.

\section{THE KNUTH SEMI-FIELDS}

In this section we consider the last three classes of Knuth's semifields defined in Section 1 . These semi-fields are characterized by the following two properties: (a) Two of the nuclei are equal, and (b) the semifield has dimension 2 over the nuclei of (a). The following result is then applicable.

THEOREM 4.1: Let $\mathrm{S}$ be a finite semi-field of order $\mathrm{p}^{\mathrm{s}}$, where $\mathrm{p}$ is a prime, with the following properties:

(i) Two of the nuclei of $\mathrm{S}$ are equal

(ii) $\mathrm{S}$ has dimension 2 over the nuclei of (a).

The autotopism group $\mathrm{G}$ of $\mathrm{S}$ is solvable.

PROOF: If $p=2$ the theorem follows from Theorem 3.2. Assume $p>2$ and that $G$ is non-solvable. Without loss of generality, assume that the nuclei in (i) are $\mathrm{N}_{\mathrm{m}}$ and $\mathrm{N}_{\mathrm{r}} \cdot$ By Theorem 3.4, we have $\mathrm{D}(\mathrm{S})=\mathrm{D}_{\mathrm{m}}=\mathrm{D}_{\mathrm{r}}$. Applying Theorem 3.4 with $\alpha=\mathrm{m}$ and $\beta=\mathrm{r}$ gives $\left.\mathrm{D}(\mathrm{S}) /<\sigma_{\mathrm{m}}\right\rangle=\operatorname{PSL}(2,5) \otimes \mathrm{z}_{2}=\operatorname{SL}(2,5), \mathrm{a}$ contradiction. Thus, for $\mathrm{p}>2$ the group $\mathrm{G}$ is solvable. 
COROLLARY 4.1.1: If $\mathrm{S}$ is a finite semi-field of dimension 2 over two of its nuclei then the autotopism group $G$ of $S$ is solvable.

PROOF: If $G$ is non-solvable then Corollary 3.4.1 implies that the three nuclei of $\mathrm{S}$ are equal. Theorem 4.1 now implies $\mathrm{G}$ is solvable. This contradiction implies that $G$ is solvable.

COROLLARY 4.1.2: If $S$ is a finite Knuth semi-field of type $K(\ell), K(r)$, or $\mathrm{K}(\mathrm{m})$ having order $\mathrm{p}^{\mathrm{s}}=\mathrm{p}^{2 \mathrm{t}}$ then the autotopism group $\mathrm{G}$ of $\mathrm{S}$ is solvable and $G$ has the following normal series

$$
G \unrhd \mathrm{L} \unrhd \mathrm{D} \unrhd \overline{\mathrm{D}} \underline{\square} 1
$$

with $|G / L|$ dividing $t$, both $|L / D|$ and $|D / D|$ dividing $p^{t}-1$, and the group $\bar{D}$ a solvable subgroup of $\operatorname{SL}\left(2, \mathrm{p}^{\mathrm{t}}\right)$ having no p-elements. Thus $|\mathrm{G}|$ divides $t\left(p^{t}-1\right)^{2} k$ where either $k=24$ or 48 or $k$ divides $2\left(p^{t} \pm 1\right)$.

PROOF: The fact that $G$ is solvable follows from Theorem 4.1. The existence of the normal series (4.1) follows from the appropriate version of Theorem 3.3. (See the Remark after Theorem 3.3.) If $\mathrm{p}=2$ then a derivation similar to that in the proof of Theorem 3.3 gives the normal series (4.1).

\section{THE INVARIANT $u$}

In [10] the following problem was investigated: Given a finite semifield plane $\mathfrak{U}$ coordinatized by the semi-field $\mathrm{S}$ with autotopism group $\mathrm{G}$, let $u=u(\mathfrak{H})$ be the number of orbits of $G$ not on one of the three axes of $\mathscr{U}$. What is the lower bound for $u$ ? In [9] it was proven that $u=1$ if and only if is desarguesian (i.e., $S$ is a field). In [10] the authors proved that for nondesarguesian semi-field planes $u \geq 5$ when $G$ is solvable and the order of $S$ is not $2^{6}$. This latter condition holds if $s$ has non-square order (i.e., if $s \neq 2$ ) or if $\mathrm{S}$ has odd dimension over one of its nuclei. (See Theorem 8.18 in [7] and Ganley [2].) We show now that $\mathrm{u} \geq 5$ if $\mathrm{S}$ has dimension 2 over one of its nuclei. In [10] it was shown that $u \leq 4$ implies that the integer $|G|$ is divisible 
by either $p^{s}-1$ or $\frac{1}{4}\left(p^{s}-1\right)$ where $p^{s}$ is the order of $s$. We show first that this implies $G$ is solvable when $S$ has dimension 2 over one of its nuclei.

THEOREM 5.1: Let $\mathrm{S}$ be a finite semi-field of order $\mathrm{p}^{\mathrm{S}}$ and having dimension 2 over one of its nuclei, and let $G$ be the autotopism group. If $|G|$ is divisible by either $p^{s}-1$ or $\frac{1}{4}\left(p^{s}-1\right)$, then $G$ is solvable.

PROOF: Without loss of generality, we may assume $\mathrm{S}$ has dimension 2 over its middle nucleus $N_{m}=G F\left(p^{t} m\right)$. Assume $G$ is non-solvable. Then $s \geq 4$. (See [11; p. 208] and [7; Theorem 8.18].) By Theorem 3.3(i) the integer $|G|$ divides $60 s\left(p^{t} m-1\right)\left(p^{t} r-1\right)$, where $N_{r}=G F\left(p^{t} r\right)$, and $p \geq 7$. Since $s \geq 4$ the integer $p^{s}-1$ has a prime division $v$ that does not divide $p^{a}-1$ for any positive integer a less than s. (See [4; Theorems 3.3, 3.5, and 3.9].) By hypothesis $v|| G \mid$; thus $v \mid 60$. (The prime $v$ does not divide $s$; because if $v \mid s$ and $v \mid\left(p^{s}-1\right)$ then $\left.v \mid p^{\frac{s}{r}}-1\right)$ since $b^{v} \equiv b(\bmod v)$ for all integers $b \geq 1$.) Since $s \geq 4$ and $p^{2} \equiv 1$ mod 3) for all $p>3$, we must have $v=5$. Hence the prime division $v$ is unique; also, if $5^{i}$ is the highest power of 5 dividing $p^{s}-1$ then we must have $i=1$. Since $p^{4} \equiv 1(\bmod 5)$ for all primes, it follows that $s=4$. Theorem 3.5 and 3.9 in [4] imply that $p=3$, contradicting the fact that $p \geq 7$. Hence $G$ is solvable.

COROLLARY 5.1.1: Let $\mathcal{U}$ be a finite semi-field plane coordinatized by a semi-field $S$ of order $p^{s}$ with $p^{s} \neq 2^{6}$. If $s$ has dimension 2 over one of its nuclei, then $\mu(2) \geq 5$.

PROOF: Assume $u\left(20 \leq 4\right.$. It follows from [10] that either $\left(p^{s}-1\right)$ or $\frac{1}{4}(p s-1)$ divides $|G|$, where $G$ is the autotopism group of $s$. Theorem 5.1 implies $G$ is solvable. Theorem 6.3 of [10] implies $u \geq 5$, a contradiction. Hence $u \geq 5$.

REMARK: The invariant $u$ also has the interpretation that it is the number of pairwise non-isomorphic semi-fields isotopic to the semi-field $S$. The 
semi-field of order 16 and dimension 2 over its kernel has exactly five nonisomorphic isotopic images.

ACKNOWLEDGMENT: The second author was supported in part by the National Science Foundation.

\section{REFERENCES}

1. Foulser, D. A. Baer p-elements in translation planes, J. Algebra 31 (1974) 354-366.

2. Ganley, M. J. Baer involutions in semi-field planes of even order, Geom. Dedi. 2(1974) 499-508.

3. Hal1, Marsha11 and J. K. Senior. The Groups of $\operatorname{Order} 2^{n}(N \geq 6)$. Macmillan, New York, 1964.

4. Hering, C. Transitive linear groups and linear groups which contain irreducible subgroups of prime order, Geom. Dedi. 2(1974) 425-460.

5. Hughes, D. R. Collineation groups of non-desarguesian planes II. Some semi-nuclear division algebras, Amer. J. Math. 82 (1960) 113-119.

6. Hughes, D. R. and E. Kleinfeld, Semi-nuclear extensions of Galois fields, Amer. J. Math. 82(1960) 389-392.

7. Hughes, D. R. and F. Piper. Projective Planes, Springer-Verlag, Berlin-Heidelberg-New York, 1973.

8. Huppert, B. Endliche Gruppen I, Springer-Verlag, Berlin-HeidelbergNew York, 1976.

9. Kallaher, M. J. A conjecture on semi-field planes, Archiv der Math. 26 (1975) 436-440.

10. Kallaher, M. J. and R. A. Liebler. A conjecture on semi-field planes II, Geom. Dedi, to appear.

11. Knuth, D. E. Finite Semi-fields and projective planes, J. Algebra 2(1965) 182-2ī. 


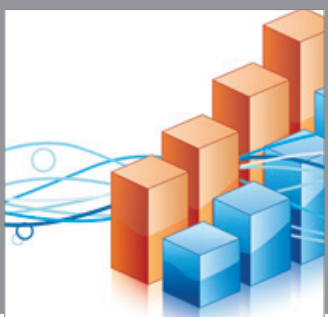

Advances in

Operations Research

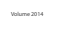

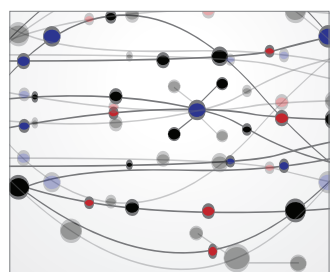

\section{The Scientific} World Journal
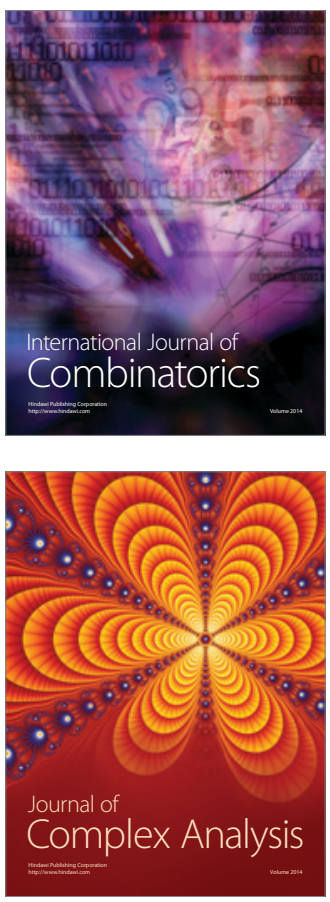

International Journal of

Mathematics and

Mathematical

Sciences
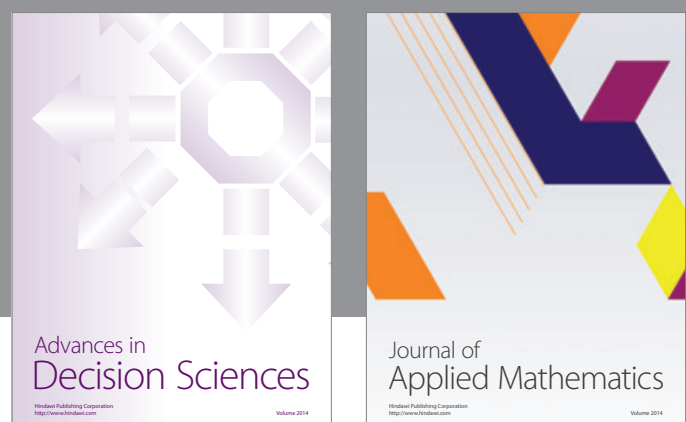

Journal of

Applied Mathematics
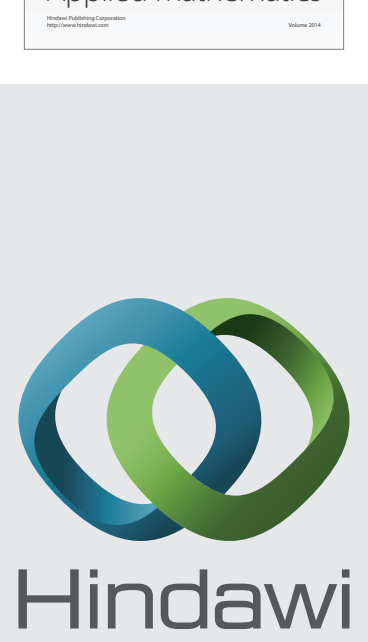

Submit your manuscripts at http://www.hindawi.com
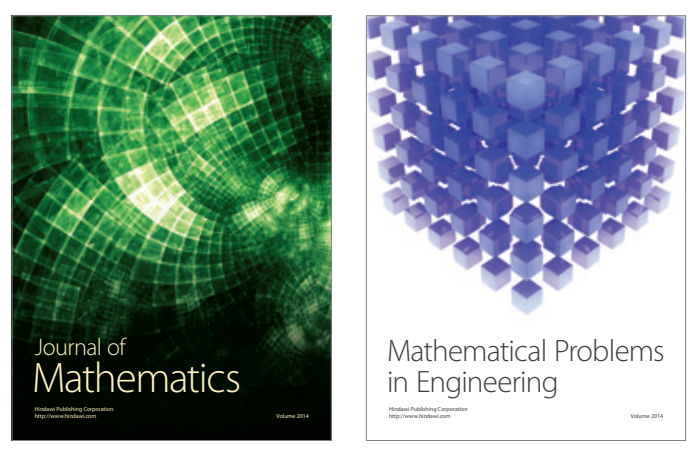

Mathematical Problems in Engineering
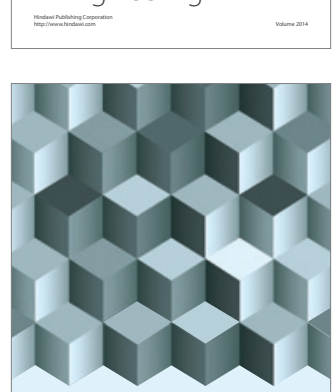

Journal of

Function Spaces
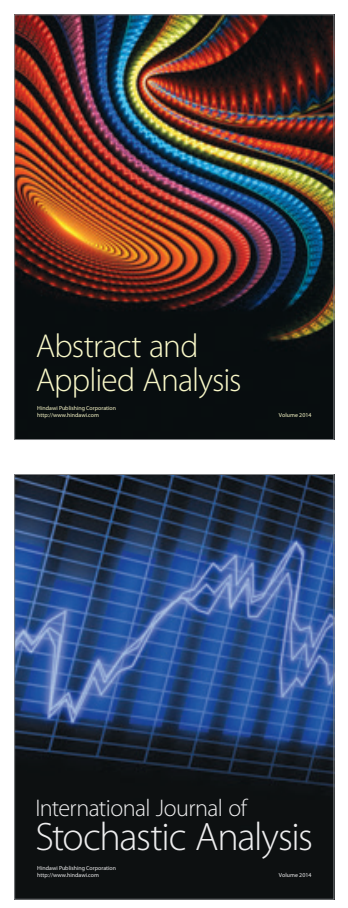

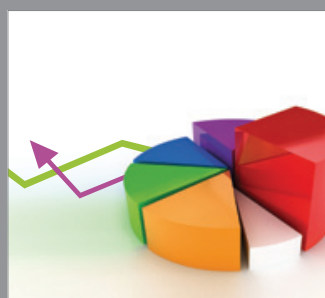

ournal of

Probability and Statistics

Promensencen
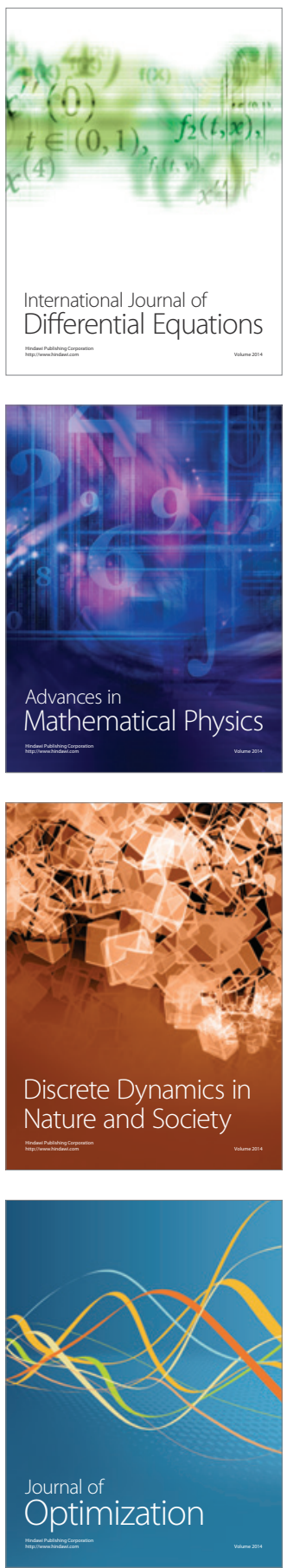